\title{
III-V SEMICONDUCTING NITRIDES ENERGY GAP UNDER PRESSURE
}

\author{
P. Perlin, I. Gorczyca, H. Teisseyre, T. Suski, E. Litwin-Staszewska, \\ S. Porowski, I. Grzegory
}

High Pressure Research Center "Unipress", Sokolowska 29/37, 01-142 Warszawa, Poland

AND N.E. Christensen

Institute of Physics, Aarhus University, 8000 Aarh us, Denmark

In this paper we present overview of our recent experimental and theoretical results concerning electronic band structure of III-V nitrides under pressure. It is shown here that the pressure coefficients of the direct gap for studied nitrides are surprisingly small. To describe tendency in changes of the gap with pressure we use a simple empirical relation.

PACS numbers: 71.25.Tn

\section{Introduction}

Nitrides of group-III metals, namely GaN, AIN, BN, InN, are characterized by very short, strong atomic bonds and relatively large ionicity. Because of that, they have many physical properties different from other III-V semiconductors. In particular, it concerns the magnitude of energy gaps and their pressure coefficients. In this paper we present the results of the pressure variation of the direct energy gap, $\mathrm{d} E_{\mathrm{g}} / \mathrm{d} p$, measured for $\mathrm{GaN}$ and $\mathrm{InN}$ as well as those calculated for the four mentioned above nitrides. We find that first principle calculations reproduce experimental results very well. Comparison with other III-V compounds shows that nitrides possess much smaller $\mathrm{d} E_{\mathrm{g}} / \mathrm{d} p$ values and in addition it appears that neither the Phillips ionicity nor the bond length can be used as a single parameter describing the discussed behavior.

\section{Experiment}

Optical absorption measurements were performed with the use of diamond anvil cell on the $\mathrm{GaN}$ and $\mathrm{InN}$ crystals. The samples were grown by means of special high-pressure, high-temperature method [1]. Pressure variation of the energy gap for the bulk semiconductors was determined from the shift of the absorption edge (see Table). Details of the experimental setup are published elsewhere [2]. 
TABLE

Pressure coefficient of direct gaps (in meV/kbar). Here $f_{\mathrm{i}}$ is Phillips ionicity (the data are taken from V. Vechten [3] and Christensen [4]) and $d$ is the bond length (in $\AA$ ).

\begin{tabular}{l|c|c|c|c}
\hline \hline Compound & \multicolumn{2}{|c|}{$\mathrm{d} E_{\mathrm{g}} / \mathrm{d} p$} & $f_{\mathrm{i}}$ & $d$ \\
\cline { 2 - 3 } & Exp. & Theor. & & \\
\hline $\mathrm{GaN}$ & 4.7 & 4.1 & 0.43 & 1.95 \\
$\mathrm{AlN}$ & - & 3.6 & 0.50 & 1.89 \\
$\mathrm{InN}$ & 2.0 & 2.5 & 0.57 & 2.16 \\
$\mathrm{BN}$ & - & 1.2 & 0.38 & 1.56
\end{tabular}

\section{Theory}

The band structures of nitrides were calculated from first principles with the use of Linear Muffin Tin Orbital method, within Local Density Approximation [2]. Band structure of the four nitrides was calculated for various pressure values and thus pressure coefficients of direct gap were determined (Table).

\section{Discussion}

The parameters commonly used in a discussion of trends in behavior of various semiconductors are Phillips ionicity [3], and bond length (reflecting the strength of interatomic interactions). It is expected accordingly that the $\mathrm{d} E_{\mathrm{g}} / \mathrm{d} p$ is smaller for more ionic compounds and for those characterized by shorter bonds. Pressure coefficients of the direct gap for the four considered nitrides show very small magnitude. It is even smaller than $\mathrm{d} E_{\mathrm{g}} / \mathrm{d} p$ observed in II-VI semiconductors which are characterized by higher Phillips ionicity with respect to the studied nitrides. The obtained coefficients are of the order of $\mathrm{d} E_{\mathrm{g}} / \mathrm{d} p$ found for diamond $(\approx 1 \mathrm{meV} / \mathrm{kbar})$.

Furthermore, it can be easily seen from Table that the pressure variation of $E_{\mathrm{g}}$ 's for the considered nitrides cannot be correlated either with Phillips ionicities or bond lengths. However, if we take into account both parameters simultaneously in the form of their ratio (see Fig. 1), it becomes possible to reproduce the tendency in pressure variation of $E_{\mathrm{g}}$ for a wide class of $\mathrm{III}-\mathrm{V}$ semiconductors. We believe that this empirical procedure is more useful than the one proposed recently by Adachi [5] who also anticipated a decrease in $\mathrm{d} E_{\mathrm{g}} / \mathrm{d} p$ with increasing ionicity in various III-V semiconductors. However, there is a need for understanding the implications of the discussed relation on the microscopic ground. 


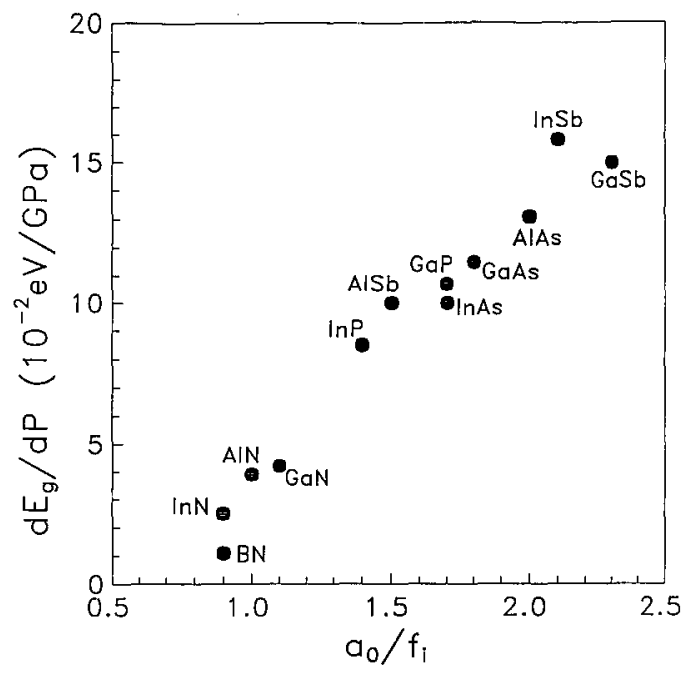

Fig. 1. Pressure coefficient of III-V nitrides in function of ratio of $a_{0}$ (lattice constant) and $f_{i}$ (Phillips ionicity).

\section{References}

[1] S. Porowski, I. Grzegory, J. Jun, in: High Pressure Chemical Synthesis, Elsevier, Amsterdam 1989.

[2] P. Perlin, I. Gorczyca, N.E. Christensen, H. Teisseyre, T. Suski, Phys. Rev. B, to be published.

[3] J.A. V. Vechten, Phys. Rev. 182, 891 (1969).

[4] N.E. Christensen, S. Sapathy, Z. Pawlowska, Phys. Rev. B 36, 1932 (1987).

[5] S. Adachi, J. Appl. Phys. 58, R1 (1985). 